\title{
Ultrafast Phenomena in Freestanding LT-GaAs Devices
}

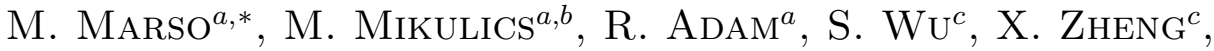 \\ I. $\mathrm{Camara}^{b}, \mathrm{~F} \cdot \mathrm{Siebe}^{b}, \mathrm{~A} . \mathrm{FörsteR}^{a}, \mathrm{R} . \mathrm{GǗsten}^{b}$, \\ P. KORDOS̆ $\check{S}^{a}$ AND R. SOBOLEWSKI ${ }^{c}$ \\ ${ }^{a}$ Institute of Thin Films and Interfaces, Research Center Jülich \\ 52425 Jülich, Germany \\ ${ }^{b}$ Max-Planck-Institut für Radioastronomie, 53121 Bonn, Germany \\ ${ }^{c}$ Department of Electrical and Computer Engineering and Laboratory \\ for Laser Energetics \\ University of Rochester, Rochester, NY 14627-0231, USA
}

We report on the fabrication and high-frequency performance of our photodetectors and photomixers based on freestanding low-temperature-grown GaAs. The MBE-grown low-temperature GaAs layers are lifted from the native GaAs substrate and transferred on top of variety of host substrates. The freestanding devices exhibit breakdown electrical fields above $200 \mathrm{kV} / \mathrm{cm}$ and dark currents below $3 \times 10^{-7} \mathrm{~A}$ at $100 \mathrm{~V}$ bias. Device photoresponse shows 0.55 ps wide electrical transients with voltage amplitudes up to $1.3 \mathrm{~V}$, measured using an electro-optical sampling technique with 100 fs wide laser pulses. Photomixing experiments at $460 \mathrm{GHz}$ yield a 9 times higher output power for the freestanding device on $\mathrm{Si} / \mathrm{SiO}_{2}$ host substrate compared to the native substrate.

PACS numbers: 72.20.Jv, 72.40.+w, 78.30.Fs, 85.60.--q, 85.60.Gz

\section{Introduction}

Low-temperature-grown GaAs (LT-GaAs) thin films exhibit subpicosecond carrier lifetime $(\sim 200 \mathrm{fs})[1]$, high resistivity $\left(>10^{7} \Omega \mathrm{cm}\right)[2]$, high breakdown electric field $(>300 \mathrm{kV} / \mathrm{cm})[2]$, and relatively large mobility $\left(\sim 150 \mathrm{~cm}^{2} /(\mathrm{V} \mathrm{s})\right)[3]$. These properties make LT-GaAs the material of choice for fast and sensitive photodetectors [4-14] and photomixers [15-20]. Typically, LT-GaAs films are grown by molecular beam epitaxy in the temperature range from $200^{\circ} \mathrm{C}$ to $300^{\circ} \mathrm{C}$, followed by rapid thermal annealing at $600^{\circ} \mathrm{C}[3,6]$. Artificially created deep-level

*corresponding author; e-mail: m.marso@fz-juelich.de 
traps are responsible for the very short carrier lifetime in LT-GaAs based opto-electronic devices. LT-GaAs based photoconductors (PC) and photomixers emerge as devices in high demand for optical communication and for generation of high-power terahertz radiation. Electrical pulses in pico- and femtosecond region are being generated by pulsed laser illumination of a metal-semiconductor-metal (MSM) structure fabricated on top of the LT-GaAs surface [1]. Using opticalheterodyne conversion in LT-GaAs photomixing, several groups obtained impressive results with output power up to $3 \mu \mathrm{W}$ at $460 \mathrm{GHz}$ or $100 \mathrm{nW}$ at $2 \mathrm{THz}$ generated radiation [15-19]. Possible further improvement of photomixing output power is related to improved thermal dissipation by integration on substrates with high thermal conductivity [20]. Generally speaking the low thermal conductivity, the same bandgap as LT-GaAs, and the high costs of the GaAs substrate make a substrate desirable with properties that are more compatible with the designated applications. Integration of LT-GaAs into hybrid circuitry using flip-chip techniques or wire bonding has been used before, but such methods reduce the intrinsic multi-gigahertz bandwidth of the devices. A possible solution is an integration of (LT-) GaAs with a substrate using thin film epitaxial lift-off employing surface bonding through van der Waals forces. For successful LT-GaAs integration into practical electronic or optoelectronic circuits however, there is not only the need for a robust technique for a reliable LT-GaAs lift-off, transfer, and bonding, but also the size of the transferred structure must be microscopic, comparable with the size of the circuits components.

The aim of this work is to investigate fabrication and properties of hybrid devices consisting of $\mathrm{SiO}_{2} / \mathrm{Si}$ or plastic substrates, integrated with micrometer-sized LT-GaAs that was epitaxially lifted and bonded at a predetermined substrate position. The use of $\mathrm{Si}$ as host substrate has strong practical implications, since $\mathrm{Si}$ is the most widely used semiconductor in electronics. In addition, Si has a higher thermal conductivity $\left(\alpha_{\mathrm{Si}} \approx 1.60 \mathrm{~W} / \mathrm{cm} / \mathrm{K}\right)$ at room temperature than GaAs $\left(\alpha_{\mathrm{GaAs}} \approx 0.59 \mathrm{~W} / \mathrm{cm} / \mathrm{K}\right)$, so using $\mathrm{Si}$ as the substrate should improve the high-power performance of optoelectronic devices, by enabling them to operate at higher excitation optical powers. Polyethylene terephthalate (PET) is an extremely interesting substrate due to its flexibility, high surface resistivity reaching $\sim 10^{13} \Omega$ /sq, optical transparency and chemical stability [21-24]. Its integration with ultrafast photoswitches and photodetectors is expected to lead to the range of applications requiring high performance, low cost on-chip generators of terahertz radiation.

\section{Fabrication}

Both our photodetectors and photomixers are based on the freestanding LT-GaAs film. The LT-GaAs layers were fabricated using a commercial Varian Mod GEN II MBE system. We started with a $300 \mathrm{~nm}$ thick layer of AlAs on top of the GaAs substrate, followed by a $500 \mathrm{~nm}$ to $1.5 \mu \mathrm{m}$ thick LT-GaAs film grown in 
the temperature range of 200 to $300^{\circ} \mathrm{C}$ with a growth rate of about $1 \mu \mathrm{m} / \mathrm{h}[3,6]$. After the MBE deposition, wafers with LT-GaAs layers were annealed in situ at $600^{\circ} \mathrm{C}$ for 10 min under local As overpressure. The AlAs/LT-GaAs bilayer was subsequently patterned by photolithography and ion-beam etching to form a set of LT-GaAs microswitches, featuring sizes from $10 \times 10 \mu \mathrm{m}^{2}$ to $150 \times 150 \mu \mathrm{m}^{2}$ [20-26]. The LT-GaAs devices were next lifted from the GaAs substrate by selective chemical etching of the AlAs layer in diluted HF solution. After cleaning in de-ionized water, a selected microswitch was transferred on top of a chosen substrate using a metallic tip, electrostatically charged and electrically isolated from the ground [20]. The release process was achieved by using a second metallic tip, which was connected to ground, thus, providing a route for the charge release after the devices were placed at their designated positions, where they were fixed by van der Waals attraction [27, 28]. To minimize the height difference between the switch and the substrate surfaces, the host substrates contained $500 \mathrm{~nm}$ deep, ion-etched "wells" matching the microswitch, pre-positioned at the designated position. Following the microswitch transfer, continuous coplanar lines crossing the devices were fabricated using $\mathrm{Ti} / \mathrm{Au}$ deposition and a standard lift-off technique. A micrograph of one of our devices is shown in Fig. 1. For photomixer fabrication a set of MSM
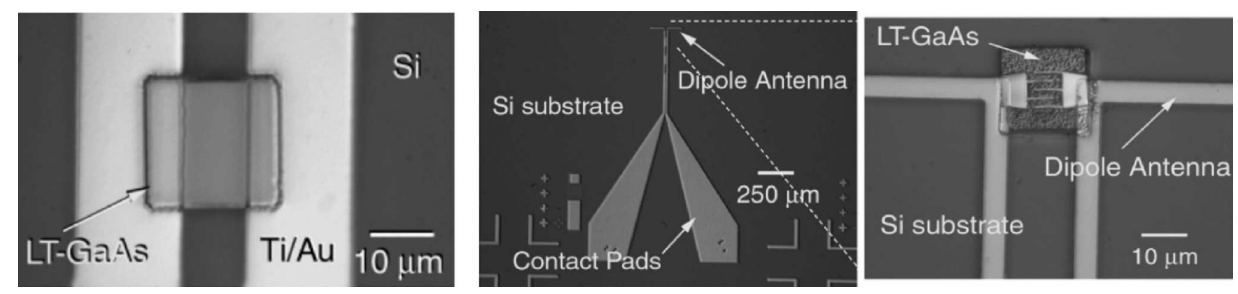

Fig. 1. Photograph of an Au/Ti CPS transmission line with a $20 \times 20 \mu \mathrm{m}^{2}$ LT-GaAs microswitch placed in the CPS gap (left). Microscope picture of the photomixer fabricated on the silicon substrate integrated with the freestanding $20 \times 20 \mu \mathrm{m}^{2}$ LT-GaAs layer, $8 \times 8 \mu \mathrm{m}^{2}$ LT-GaAs/MSM interdigitated structure integrated with $460 \mathrm{GHz}$ dipole antenna (middle, right).

interdigitated finger structures was prepared on top of the LT-GaAs using conventional lift-off process, prior to the ion-beam etching and epitaxial lift-off from the native substrate. Finger width and spacing of the MSM structure were typically $200 \mathrm{~nm}$ and $1.8 \mu \mathrm{m}$, respectively. Following the transfer of LT- -GaAs on the top of a chosen substrate, conventional lift-off was used to connect LT-GaAs/MSM with $460 \mathrm{GHz}$ tuned single dipole antenna and with coplanar lines containing low-pas filters and serving to provide dc bias leads. Figure 1 shows the micrograph of an LT-GaAs/MSM photomixer layout with interdigitated structure integrated with a $460 \mathrm{GHz}$ dipole antenna, respectively. 


\section{Results and discussions}

Figure 2 shows typical current-voltage $(I-V)$ characteristics of freestanding LT-GaAs transferred on top of a sapphire, $\mathrm{Si} / \mathrm{SiO}_{2}$, and PET plastic substrates [20], as well as for as-grown LT-GaAs on native GaAs substrate, measured in the dark. The as-grown samples exhibit ohmic dependence up to $\sim 10 \mathrm{~V}$ and at higher bias nonlinear behavior is observed. On the other hand, the freestanding devices transferred on top of a host substrate show ohmic behavior in the whole range of applied voltages up to $200 \mathrm{~V}$ (corresponding to an average electric field of $200 \mathrm{kV} / \mathrm{cm}$ ). Furthermore, Fig. 2 demonstrates that our freestanding structures exhibit more than 10-fold decrease of the dark current as compared to the as-grown devices, and they show no sign of electrical breakdown up to $200 \mathrm{~V}$ of dc bias. This improvement shows the advantage of the insulating host substrates compared to the semi-insulating GaAs native substrate.
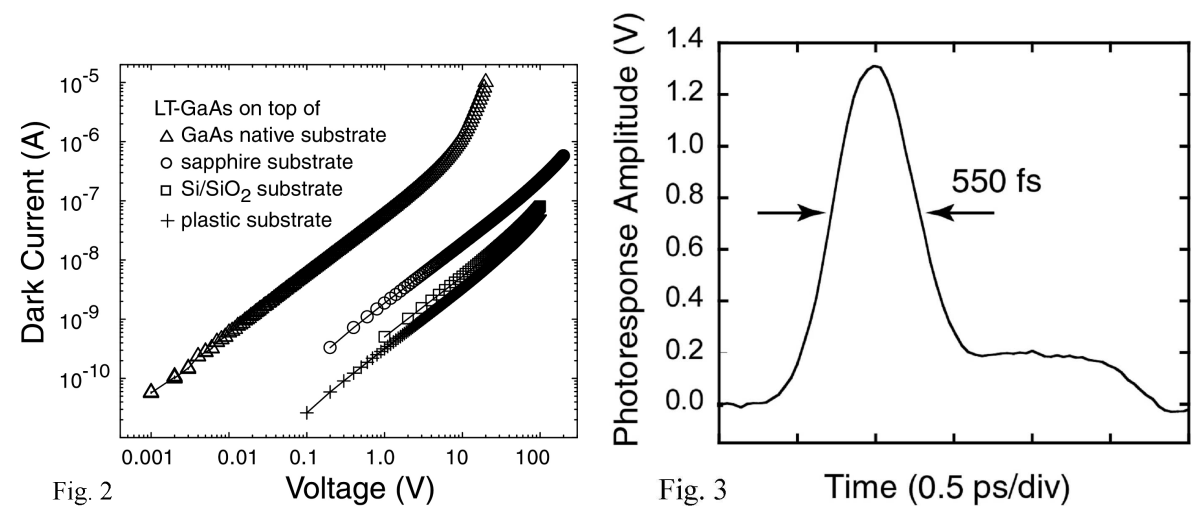

Fig. 2. $\quad I-V$ characteristics of an as-grown LT-GaAs-on-GaAs device and the LT-GaAs microswitch transferred on top of the sapphire, $\mathrm{Si} / \mathrm{SiO}_{2}$, and PET plastic substrates.

Fig. 3. 550 fs FWHM photoresponse of a freestanding LT-GaAs switch exposed to $100 \mathrm{fs}$ wide, $810 \mathrm{~nm}$ wavelength optical pulses, at $92 \mathrm{~V}$ bias and $2 \mathrm{~mW}$ input power.

The time-resolved photoresponse of the freestanding LT-GaAs microswitches was measured using electro-optic sampling [29-31] with 100 fs wide optical pulses from a Ti:sapphire laser at $810 \mathrm{~nm}$ wavelength. The transients were sampled with the spot located only $\sim 50 \mu \mathrm{m}$ away from the device. Thus, the signal distortion caused by pulse dispersion effects in the coplanar lines was minimized enabling the measurement of near-to-intrinsic carrier dynamics in the LT-GaAs films. The time-resolved photoresponse signal of a freestanding LT-GaAs switch transferred on the $\mathrm{Si} / \mathrm{SiO}_{2}$ substrate and illuminated by $810 \mathrm{~nm}$ photons is shown in Fig. 3. The transient exhibits a 550 fs full-width-at-half-maximum (FWHM) photoresponse, that is nearly identical to a previously measured value of 580 fs for an LT-GaAs photodetector on its native GaAs substrate [1]. 
The photoresponse amplitude increases linearly with increasing photoexcitation power $P_{\text {ex }}$. Above $2 \mathrm{~mW}$, however, the signal amplitude starts to level off. We believe that the saturation arises due to the fact that at a threshold $P_{\text {ex }}$ all carriers for a given photon energy are excited which leads to the so-called band filling effect. When the free carrier density becomes too high, space-charge effect can contribute to the onset of saturation by affecting the carrier mobility in the conduction band. The decrease of the carrier collection efficiency can also be illustrated by measuring the dc responsivity that is defined as the ratio of the photocurrent and the input optical power. The voltage responsivity measured at a bias of $30 \mathrm{~V}$ decreases from $25 \mathrm{~V} / \mathrm{W}$ at $1 \mathrm{~mW}$ to $10 \mathrm{~V} / \mathrm{W}$ at $7 \mathrm{~mW}$ optical input power [20].

Figure 4 shows the response amplitude and response time as a function of applied bias, measured at the excitation power of $\sim 0.46 \mathrm{~mW}$. The pulse amplitude increases linearly at low voltages, and at higher biases the amplitude saturates, indicating a decrease of carrier collection efficiency. Closed circles illustrate constant photoresponse time of our devices for biases up to $120 \mathrm{~V}$. For comparison, open circles indicate data from Ref. [32] performed on an LT-GaAs switch on native GaAs substrate. The considerable increase of the response time for high electric fields, in contrast to the freestanding device, can be explained by thermal effects due to the lower thermal conductivity of GaAs [14] or by the penetration of the electric field into the GaAs substrate where slow carriers can be excited [20].

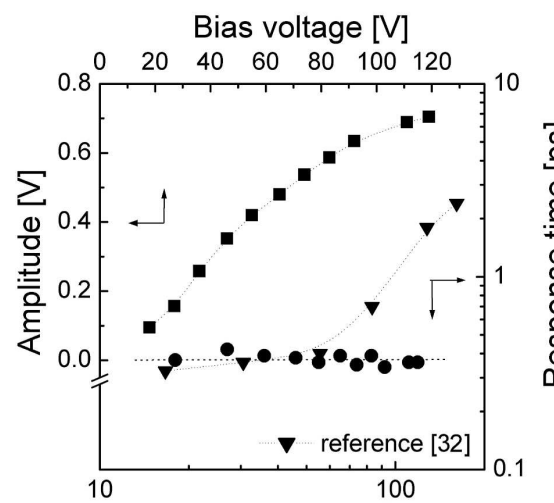

Fig. 4 Average electric field $[\mathrm{kV} / \mathrm{cm}]$

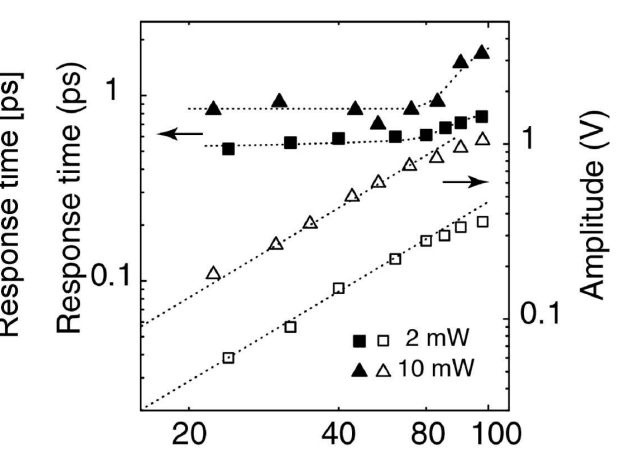

Fig. 5

Voltage (V)

Fig. 4. Response time as a function of an average electric field (right and bottom axes) and amplitude as a function of applied bias voltage (left and upper axes) for LT-GaAs-on-Si microswitch. Curve marked by triangles shows response times for LT-GaAs-on-GaAs given in [32].

Fig. 5. Response time and amplitude of electrical transients of the LT-GaAs-on-PET photodetector as a function of bias voltage for 2 and $10 \mathrm{~mW}$ optical power. The dotted lines are guides for eye. 
The LT-GaAs-on-PET devices were first characterized by $I-V$ measurements in the dark and under the $850 \mathrm{~nm}$ continuous-wave illumination. The $I-V$ curves exhibit ohmic behavior up to $70 \mathrm{~V}$. The space-charge effect is not observed, which indicates efficient suppression of high-field region near the contacts. The devices exhibit low dark currents of $6 \times 10^{-9} \mathrm{~A}$ at $10 \mathrm{~V}$ and $\leq 10^{-7} \mathrm{~A}$ at $100 \mathrm{~V}$, and show breakdown voltages in the range from $110 \mathrm{~V}$ to $130 \mathrm{~V}$. The results from electro-optic sampling measurements yield 0.7 ps FWHM response time, comparable to reported values on freestanding LT-GaAs on $\mathrm{Si} / \mathrm{SiO}_{2}$ [28]. Device response time and amplitude as a function of applied bias voltage are shown in Fig. 5. Response times down to $0.55 \mathrm{ps}$ and amplitudes up to $1 \mathrm{~V}$ have been achieved [33]. In the low voltage bias region the response time is constant and the amplitude increases proportionally with the bias, as one would expect. On the other hand, the response time increases and the amplitude saturates if the bias voltage is increased above 70-75 V. This indicates the influence of heating effects at higher biases that become apparent due to the very low thermal conductivity of PET substrate $\left(1.5 \mathrm{~mW} \mathrm{~cm}^{-1} \mathrm{~K}^{-1}\right)$. Measurements at increased illumination power confirm the above result that heating effects do not play a significant role at biases below 70-75 V. However, for $97 \mathrm{~V}$ bias voltage and $10 \mathrm{~mW}$ input power an increase of the response time reached 1.7 ps thus supporting the effect explanation by overheating.

Photomixer experiments were performed by an experimental setup with two tunable semiconductor lasers operated at $780 \mathrm{~nm}$ wavelength that are coupled to an optical amplifier. A part of the amplifier output signal is monitored by a wavemeter to determine the difference frequency between the two lasers. The rest is coupled to a monomode fiber optic and directed to the photodetector area of the photomixer. The applied bias generates a photocurrent that is modulated with the frequency difference of the two lasers. A Golay cell allows the measurement of the power of the generated radiation that is emitted by the dipole antenna. The above-mentioned improvements in thermal management of our freestanding photoconducting devices should lead to an increase of the photomixer output power. One example of the working device is shown in Fig. 1 showing the photomixer device with $20 \times 20 \mu \mathrm{m}^{2}$ LT-GaAs layer with thickness about $1 \mu \mathrm{m}$ on the silicon substrate coupled with dipole antenna. We used silicon substrate because of its 3-times higher thermal conductivity in comparison with the native GaAs substrate. As shown in Fig. 6, at low photomixer bias, we observed output power exceeding 9-times the output power of the devices fabricated on native GaAs substrate [34]. We ascribe the improvement to the better thermal management of the device. At higher voltages, corresponding to the approximately $12 \mathrm{kV} / \mathrm{cm}$ average electric field, however, electrical breakdown occurred due to not fully optimized electrical contact in the LT-GaAs-metallization interface. We believe that improvements in device fabrication technique will lead to future increase of the device output exceeding the current in best devices on native GaAs. 


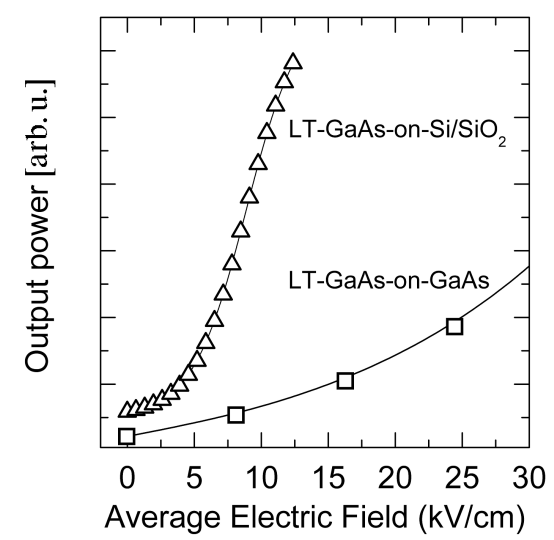

Fig. 6. Comparison of output power at $460 \mathrm{GHz}$ from photomixers based on LT-GaAs on native GaAs and LT-GaAs on silicon substrates.

\section{Conclusions}

We selectively etched $20 \times 20 \mu \mathrm{m}^{2}$ LT-GaAs thin-film square pieces and transferred them on top of $\mathrm{Si} / \mathrm{SiO}_{2}$, sapphire, and plastic (polyethylene terephthalate) substrates. The thin films were subsequently integrated with metallic transmission lines, forming freestanding photoconductive microswitches and highfrequency photomixers. The freestanding devices exhibited 10-fold lower dark currents and higher breakdown voltages as compared to devices patterned on native GaAs substrates. High saturation voltage and the linear dependence of the photoresponse amplitude on the bias voltage for both photoconductive switches and photomixing devices result from high quality contacts formed between our freestanding LT-GaAs films and the metallic electrodes. Electro-optic sampling measurements showed $0.55 \mathrm{ps}$ wide response at $810 \mathrm{~nm}$ wavelength excitation, with up to $1.3 \mathrm{~V}$ photoresponse amplitude and above $10^{3} \mathrm{~V} / \mathrm{W}$ responsivity. The output power of the freestanding LT-GaAs-on-Si photomixers was measured using two-laser heterodyne mixing at $460 \mathrm{GHz}$ and showed ninefold increase of the output power in low voltage bias region compared to the devices fabricated on top of GaAs substrates.

Our technique is also applicable for other host substrates, as substrates with large bandgap as well as with high thermal conductivity. While the latter results in more efficient device cooling, large bandgap enables device biasing at higher electric field intensities without carrier collection from the substrate. Sapphire and $\mathrm{MgO}$ are extremely attractive substrate materials suitable for cryogenic applications due to their compatibility with high-temperature superconductors. Integration of LT-GaAs with transparent substrates, such as MgO, sapphire, and PET, allows experiments in the transmission mode, e.g., optical excitation of devices through the substrate. In summary, the freestanding LT-GaAs microswitches are suitable 
for hybrid optoelectronic applications, on-chip femtosecond-pulse generators, as well as for low-cost high-power terahertz frequency generators that can be placed at any chosen position of the electronic circuit.

\section{References}

[1] P. Kordoš, A. Föster, M. Marso, F. Rüders, Electron. Lett. 34, 119 (1998).

[2] D.C. Look, Thin Solid Films 231, 61 (1993).

[3] P. Kordoš, M. Marso, A. Föster, J. Darmo, J. Betko, G. Nimtz, Appl. Phys. Lett. 71, 1118 (1997).

[4] S. Gupta, J. Whitaker, G. Mourou, IEEE J. Quantum Electron. 28, 2464 (1992).

[5] J.F. Whitaker, Mater. Sci. Eng. B 22, 61 (1993).

[6] J. Betko, M. Morvic, J. Novak, A. Förster, P. Kordoš, J. Appl. Phys. 86, 6243 (1999).

[7] S. Gupta, M.Y. Frankel, J.A. Valdmanis, J.F. Whitaker, G.A. Mourou, F.W. Smith, A.R. Calawa, Appl. Phys. Lett. 59, 3276 (1991).

[8] Y.-J. Chiu, S.B. Fleischer, D. Lasaosa, J.E. Bowers, Appl. Phys. Lett. 71, 2508 (1997).

[9] M.Y. Liu, S.Y. Chou, T.Y. Hsiang, S. Alexandrou, R. Sobolewski, J. Vac. Sci. Technol. B 10, 2832 (1992).

[10] K. Kato, IEEE Trans. Microw. Theory Tech. 47, 1265 (1999).

[11] S.Y. Chou, Y. Liu, P.B. Fischer, Appl. Phys. Lett. 61, 819 (1992).

[12] D. Krökel, D. Grischkowsky, M.B. Ketchen, Appl. Phys. Lett. 54, 1046 (1989).

[13] E. Sano, T. Shibata, Appl. Phys. Lett. 55, 2748 (1989).

[14] M.Y. Frankel, J.F. Whitaker, G.A. Mourou, F.W. Smith, A.R. Calawa, IEEE Trans. Electron Devices 37, 2493 (1990).

[15] E.R. Brown, K.A. McIntosh, K.B. Nichols, C.L. Dennis, Appl. Phys. Lett. 66, 285 (1995)

[16] A.S. Pine, R.D. Suenram, E.R. Brown, K.A. McIntosh, J. Mol. Spectrosc. 175, 37 (1996).

[17] E. Peytavit, G. Mouret, J.F. Lampin, P. Masselin, P. Mounaix, F. Mollot, D. Lippens, in: Proc. 8th Intern. Conf. on Terahertz Electronics September 2000, Darmstadt (Germany).

[18] E. Peytavit, S. Arscott, D. Lippens, G. Mouret, S. Matton, P. Masselin, R. Bocquet, J.F. Lampin, L. Desplanque, F. Mollot Appl. Phys. Lett. 81, 1174 (2002).

[19] S. Matsuura, M. Tani, K. Sakai, Appl. Phys. Lett. 70, 59 (1997).

[20] M. Mikulics, Ph.D. Thesis, Research Center Jülich, Jülich 2004.

[21] R. Paetzold, K. Heuser, D. Henseler, S. Roeger, Appl. Phys. Lett. 82, 3342 (2003).

[22] P. Kus, A. Plecenik, L. Satrapinsky, Y. Xu, R. Sobolewski, Appl. Phys. Lett. 81, 2199 (2002).

[23] Y. Ichikawa, T. Yoshida, T. Hama, H. Sakai, K. Harashima, Solar Energy Mat. Es Solar Cells 66, 107 (2001).

[24] H. Jia, J. Veldeman, M. Burgelman, J. Magn. Magn. Mater. 223, 73 (2001). 
[25] E. Yablonovitch, T. Gmitter, J.P. Harbison, R. Bhat, Appl. Phys. Lett. 51, 2222 (1987).

[26] E. Yablonovitch, D.M. Hwang, T.J. Gmitter, L.T. Florez, J.P. Harbison, Appl. Phys. Lett. 56, 2419 (1990).

[27] M. Mikulics, M. Marso, R. Adam, A. Fox, D. Buca, A. Förster, P. Kordoš, in: 2001 Intern. Symp. on Electron Devices for Microwave and Optoelectronic Applications, Vienna, November 2001, Proc. 9th EDMO, IEEE Catalog Number: 01TH8567, ISBN: 0-7803-7049-X, 155 (2001).

[28] R. Adam, M. Mikulics, A. Förster, J. Schelten, P. Kordoš, M. Siegel, X. Zheng, S. Wu, R. Sobolewski, Appl. Phys. Lett. 81, 3485 (2002).

[29] J.A. Valdmanis, G.A. Mourou, IEEE J. Quantum Electron. QE-22, 69 (1986).

[30] M.Y. Frankel, J.F. Whitaker, G.A. Mourou, J.A. Valdmanis, IEEE Microw. Guided Wave Lett. 1, 60 (1991).

[31] R. Adam, R. Sobolewski, M. Darula, Proc. SPIE 4058, 230 (2000).

[32] N. Zamdmer, Q. Hu, K.A. McIntosh, S. Verghese, Appl. Phys. Lett. 75, 2313 (1999).

[33] M. Mikulics, R. Adam, M. Marso, A. Förster, P. Kordoš, S. Wu, X. Zheng, R. Sobolewski, submitted to Appl. Phys. Lett.

[34] M. Mikulics, F. Siebe, X. Zheng, R. Adam, M. Marso, H. Stüer, R. Sobolewski, R. Güsten, P. Kordoš, in: 4th Symp. on Non-Stoichiometric III-V Compounds, October 2002, Asilomar (USA). 